\title{
Anomalous quantum confinement of the longitudinal optical phonon mode in PbSe quantum dots
}

\author{
J. Habinshuti, ${ }^{1,2}$ O. Kilian,,${ }^{1,3}$ O. Cristini-Robbe, ${ }^{4}$ A. Sashchiuk, ${ }^{5}$ A. Addad, ${ }^{6}$ S. Turrell, ${ }^{2}$ E. Lifshitz, ${ }^{5}$ \\ B. Grandidier, ${ }^{1}$ and L. Wirtz, ${ }^{1,7}$ \\ ${ }^{1}$ Institut d'Electronique, de Microélectronique et de Nanotechnologie (IEMN), CNRS, UMR 8520, Département ISEN, \\ 41 bd Vauban, 59046 Lille Cédex, France \\ ${ }^{2}$ Laboratoire de Spectrochimie Infrarouge et Raman, LASIR, Université des Sciences et Technologies de Lille, \\ Bât C5, 59655 Villeneuve d'Ascq Cedex, France \\ ${ }^{3}$ Department of Experimental Physics, Comenius University, Mlynska dolina F1, 84248 Bratislava 4, Slovakia \\ ${ }^{4}$ Laboratoire de Physique des Lasers, Atomes et Molécules (CNRS, UMR 8523), Bât P-5, Université des Sciences et Technologies de Lille, \\ 59655 Villeneuve d'Ascq Cedex, France \\ ${ }^{5}$ Schulich Faculty of Chemistry, Russell Berrie Nanotechnology Institute, Technion, Haifa 32000, Israel \\ ${ }^{6}$ Laboratoire de Structure et Propriétés de l'Etat Solide, Université des Sciences et Technologies de Lille, 59655 Villeneuve d'Ascq, France \\ ${ }^{7}$ Physics and Materials Science Research Unit, University of Luxembourg, 162 a Avenue de la Faïencerie, L-1511 Luxembourg, Luxembourg
}

(Received 14 February 2013; published 24 September 2013)

\begin{abstract}
We have investigated the diameter dependence of the Raman spectra of lead selenide nanocrystals. The first-order Raman peak at about $136 \mathrm{~cm}^{-1}$ and its second-order overtone at twice this wavenumber move up in energy with decreasing nanocrystal diameter. This anomalous behavior is interpreted in terms of quantum confinement of the longitudinal optical (LO) phonon whose frequency displays a minimum at $\Gamma$ in the dispersion of bulk PbSe. We perform ab initio calculations of the phonons of PbSe slabs with up to 15 layers. The LO mode perpendicular to the slab shifts indeed upwards with decreasing layer thickness, thus validating the interpretation of the anomalous radius dependence of the Raman spectra in terms of quantum confinement.
\end{abstract}

DOI: 10.1103/PhysRevB.88.115313

PACS number(s): 63.22.Kn, 78.67.Bf

\section{INTRODUCTION}

The lead chalcogenides $\mathrm{Pb} X(X=\mathrm{S}, \mathrm{Se}$, or Te) are unusual IV-VI semiconductors with a rock salt crystal structure and with very small direct band gaps $(410-280 \mathrm{meV}$ at room temperature $^{1}$ ) at the high-symmetry point $L$. This band gap can be reduced to zero upon compressing the lattice, ${ }^{2,3}$ and in its calculation, the effects of both spin-orbit coupling and electron correlation must be carefully taken into account. ${ }^{3,4}$ Closely related to the electronic band structure, the lattice dynamics of $\mathrm{Pb} X$ (Ref. 5) also display some unusual features, e.g., the transverse optical (TO) phonon mode displays a minimum at Gamma and is very soft $\left[60 \mathrm{~cm}^{-1}\right.$ for PbS, ${ }^{6} 47 \mathrm{~cm}^{-1}$ for $\mathrm{PbSe},{ }^{7} 30 \mathrm{~cm}^{-1}$ for PbTe (Ref. 8)], which means that the materials are close to a ferroelectric instability. ${ }^{9}$ Furthermore, the longitudinal optical (LO) mode displays an anomalous dip at $\Gamma$ which can be explained as due to a pseudo-Jahn-Teller effect ${ }^{10}$ or as a near Kohn anomaly. ${ }^{11}$

The phonon dispersion of PbS (Ref. 6) and PbTe (Ref. 8) have been measured by inelastic neutron diffraction. Only scarce neutron diffraction data is available for PbSe. ${ }^{12,13}$ Raman characterization of $\mathrm{Pb} X$ is delicate because both $\mathrm{TO}$ and LO optical modes are in principle not Raman active in the rock salt crystal structure. Nevertheless, in some Raman experiments, the LO mode (which is infrared, but a priori, not Raman active) has been observed. In PbSe, the LO mode was detected around $135 \mathrm{~cm}^{-1}$ for PbSe films on a glass substrate ${ }^{14}$ and on a (111) surface of $\mathrm{BaF}_{2} \cdot{ }^{15}$ In these cases, the observability of the LO mode may be explained by a reduction of symmetry in the thin film geometry. The results coincide well with the $133 \mathrm{~cm}^{-1}$ measured by tunneling experiments ${ }^{16}$ and the $136 \mathrm{~cm}^{-1}$ from neutron diffraction. ${ }^{12}$ Similarly, the LO mode had been detected in the first-order Raman spectrum of a $\mathrm{PbTe}$ sample coated with a thin $\mathrm{Pb}$ layer, which induces surface electric fields and energy-band bending. ${ }^{17}$ In recent Raman measurements on PbSe single crystals under resonant conditions, a peak at $130 \mathrm{~cm}^{-1}$ is found. ${ }^{18}$ The authors argue that the LO mode observation may be due to the resonant Fröhlich mechanism. ${ }^{19}$

The small band gap of lead chalcogenides, together with a large exciton diameter (e.g., $20 \mathrm{~nm}$ in $\mathrm{PbS}$ and $46 \mathrm{~nm}$ in $\mathrm{PbSe}$ ) and small effective electron and hole masses, make them an ideal candidate for strong quantum confinement in the nanocrystalline form. ${ }^{20}$ While many studies on optical absorption and luminescence spectroscopy as a function of the diameter of $\mathrm{PbX}$ quantum dots (nanocrystals) have been performed, ${ }^{21-23}$ relatively few works on the Raman spectroscopy of these materials exist, and the results are contradictory. ${ }^{24-27}$ Some results suggest a redshift of the LO-mode line in the Raman spectra of nanocrystalline $\mathrm{Pb} X$ as compared to the spectrum of bulk $\mathrm{Pb} X$, but other studies give evidence for a blueshift. Since the spectra may be strongly dependent on the experimental conditions, only a systematic Raman study of nanocrystals with different diameters under constant experimental conditions can give a definite answer. This is the purpose of our paper in which we provide such a study for spherical $\mathrm{PbSe}$ nanocrystals of varying diameters. We observe a blueshift of the LO-mode-related Raman line with decreasing crystal diameter. Our findings are interpreted in terms of a simple quantum-confinement model for the LO mode as well as by ab initio calculations of phonons in $\mathrm{PbSe}$ slabs of varying thickness.

\section{EXPERIMENTS}

$\mathrm{PbSe}$ nanocrystals (NCs) were synthesized according to literature methods. ${ }^{28}$ Briefly, lead oleate precursors were prepared from lead acetate trihydrate dissolved in a mixture 

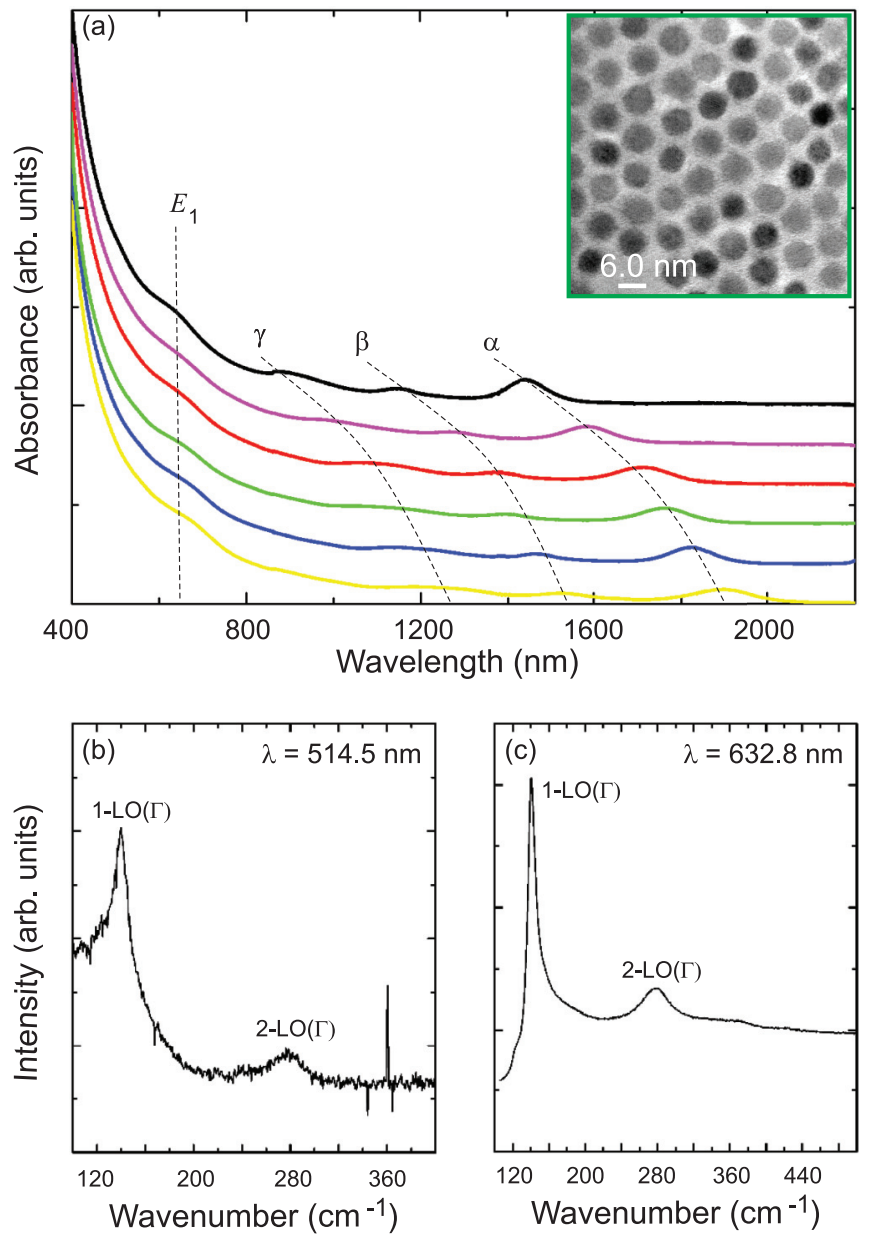

FIG. 1. (Color online) (a) Normalized absorbance spectra of PbSe nanocrystals for a range of mean diameter between $7.1 \mathrm{~nm}$ (bottom) and $4.5 \mathrm{~nm}$ (top). The optical transitions are labelled $\alpha, \beta, \gamma$, and $E_{1}$. Inset: TEM image of a monolayer of $\mathrm{PbSe}$ nanocrystals with a mean diameter of $6.0 \mathrm{~nm}$. (b) and (c) Raman spectra of PbSe nanocrystals with a mean diameter of $6.8 \mathrm{~nm}$ for two optical excitation wavelengths.

of oleic acid and 1-octadecene that was stirred at a temperature of $110^{\circ} \mathrm{C}$. Tri-n-octylphosphine selenide precursors were prepared from the dissolution of selenium in tri-n-octylphosphine. Then they were injected into the solution containing lead oleate precursors that was heated at $180^{\circ} \mathrm{C}$. The reaction was allowed to continue for 1 to $15 \mathrm{~min}$ at a temperature between 110 and $145^{\circ} \mathrm{C}$, depending on the nanocrystal size that had to be reached. After centrifugation and redispersing the precipitated nanocrystals, the suspension was thoroughly washed to remove unreacted species.

The nanocrystal size was determined from the absorbance spectrum of the nanocrystal suspension and compared with the size measured with high-resolution transmission electronic microscopy (HR-TEM). Figure 1(a) shows representative absorption spectra of six PbSe nanocrystal suspensions. The nanocrystal mean diameter varies between 4.5 and $7.0 \mathrm{~nm}$. The narrow size distribution of the nanocrystals results in sharp optical transitions that were assigned based on the nomenclature used in Ref. 21. As seen in Fig. 1(a), the lowest energy transitions $\alpha, \beta, \gamma$ are blueshifted as the nanocrystal size decreases. A higher transition, labelled $E_{1}$, is also visible. It is not affected by quantum confinement and corresponds to a transition that occurs at critical points of the $\mathrm{PbSe}$ bulk band structure. ${ }^{29,30}$ From HR-TEM measurements of the suspensions characterized in Fig. 1(a), the standard deviation for the size distribution was found to be $4 \%$. For smaller and larger nanocrystal sizes, the size distribution slightly increases.

Seven samples of $\mathrm{PbSe}$ nanocrystals with an average size ranging from 2.5 to $8.5 \mathrm{~nm}$ were then studied by Raman spectroscopy. For that experiment, solutions of nanocrystals in toluene were drop-cast on a $\mathrm{Si}$ wafer or on glass. The Raman spectra were excited by an Ar-ion laser $(514.5 \mathrm{~nm})$ or by a He-Ne laser $(632.8 \mathrm{~nm})$ at several laser powers and were analyzed by a LabRAM Dilor Jobin Yvon spectrometer or a T64000 confocal micro-Raman spectrometer. The incident light was focused into a spot diameter of $1 \mu \mathrm{m}$. Spectral resolution of the Raman spectrometer was about $1 \mathrm{~cm}^{-1}$. Each spectrum was measured with an accumulation time of 100-300 s. In order to perform micro-Raman measurements between room temperature and $250^{\circ} \mathrm{C}$, a Linkam THMS 600 heating stage was used.

Starting with the Ar-ion laser, the analysis of $\mathrm{PbSe}$ nanocrystals with a mean diameter of $6.8 \mathrm{~nm}$ reveals the existence of two peaks at 140 and $281 \mathrm{~cm}^{-1}$ that are due to the excitation of one LO phonon (1-LO peak) and two LO phonons (2-LO peak), respectively. ${ }^{26,27}$ Choosing another laser wavelength of $632.8 \mathrm{~nm}$ that is resonant with the $E_{1}$ transition yields a similar spectrum but improves the quality of the measurements. As a result, this excitation wavelength was preferred in the following experiments. While this result favors a resonant Fröhlich mechanism to account for the excitation of the LO modes in PbSe nanocrystals, the Raman activity could also be caused by a reduction of the nanocrystal symmetry, since $\mathrm{PbSe}$ nanocrystals are known to be faceted. ${ }^{31,32}$

As the laser power has been shown to induce structural changes in semiconductor nanocrystals, ${ }^{33}$ we first turn our attention to the positions of the 1-LO and 2-LO peaks as a function of the laser power. As shown in Fig. 2(a) for PbSe nanocrystals with a mean diameter of $5.5 \mathrm{~nm}$, an increase of the laser power causes a significant shift of the peak position for the 1 -LO mode. The peak decreases from 142.4 to $138.8 \mathrm{~cm}^{-1}$ as the laser power increases from 1 to $10 \mathrm{~mW}$. A similar trend is observed for PbSe powder [Fig. 2(c)] that can be considered as bulk material due to the micrometer size of the PbSe grains. Interestingly, working with the smallest laser power, as well as raising the sample temperature, both lead to a systematic redshift of the 1-LO mode [Figs. 2(b) and 2(d)] that is quite consistent with the one found when the laser power changes. Although heating the nanocrystals leads to a larger lattice and thus a reduction of the phonon wavenumber in agreement with our observation, it could also contribute to an increase of the oxidation of the nanocrystal surfaces.

Measurements were thus performed in air or in a glove box filled with nitrogen. Figure 3(a) clearly shows a redshift of the 1-LO and 2-LO modes, when the nanocrystals are exposed to air, in agreement with Ref. 34. Focusing on the 1-LO mode, the peak becomes asymmetric after air exposure, a shoulder being clearly visible at smaller wavenumbers. We attribute this shoulder to the $\mathrm{Pb}-\mathrm{O}-\mathrm{Pb}$ stretching mode. The second overtone of this mode occurs at twice the wavenumber and as a result 

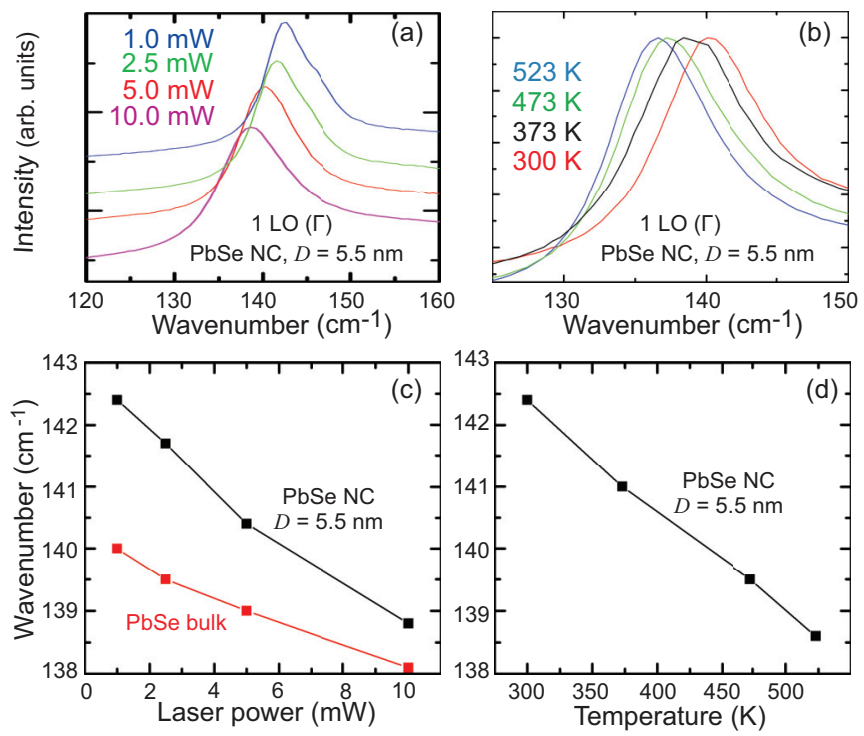

FIG. 2. (Color online) (a) Raman spectra of a 5.5-nm PbSe nanocrystal film for different laser powers. The experiment was performed at $300 \mathrm{~K}$. (b) Raman spectra of a 5.5-nm PbSe nanocrystal film for different sample temperatures. The laser power was set to $1.0 \mathrm{~mW}$. (c) Raman wavenumbers of the 1-LO peak as a function of the laser power for the 5.5-nm PbSe nanocrystal film and a bulk $\mathrm{PbSe}$ sample. (d) Raman wavenumbers of the 1-LO phonon mode as a function of the temperature for the 5.5-nm PbSe nanocrystal film.

redshifts and broadens the peak related to the 2-LO mode of $\mathrm{PbSe}$. Therefore, in order to precisely measure the energy of the phonon modes in PbSe nanocrystals with different sizes, it is necessary to avoid their exposure to air and use a low laser power to preserve the nanocrystals from any chemical or structural modifications. The subsequent experiments were thus performed at the lowest laser power of $1 \mathrm{~mW}$, limiting the nanocrystal exposition to air as much as possible.

Finally, Raman spectra were measured on different places of the same sample. As shown in Fig. 3(b) for a sample consisting of a thin film of 5.5-nm PbSe nanocrystals, small but still significant shifts are observed in the peak position of the 1-LO mode. As an increase of the heat leads to a redshift
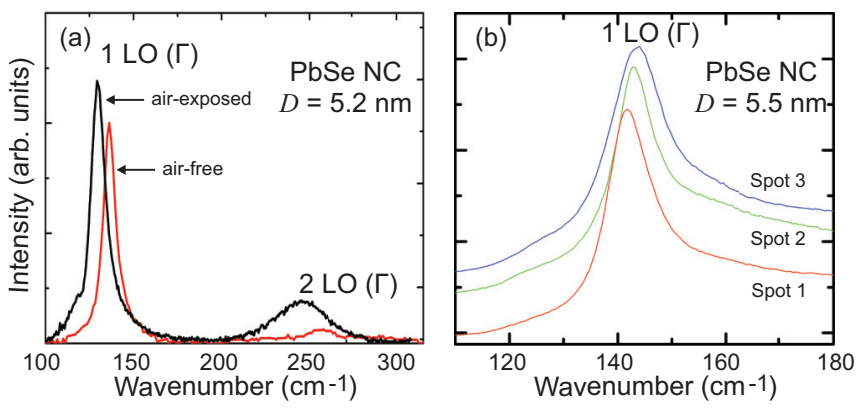

FIG. 3. (Color online) (a) Comparison of Raman spectra for a 5.2-nm PbSe nanocrystal film measured in an air-free environment (red) and air exposed (black), both excited at $5 \mathrm{~mW}$. (b) Spatial fluctuations in the position of the 1-LO peak of 5.5-nm PbSe nanocrystal films. The laser power was set to $1.0 \mathrm{~mW}$ in (b).

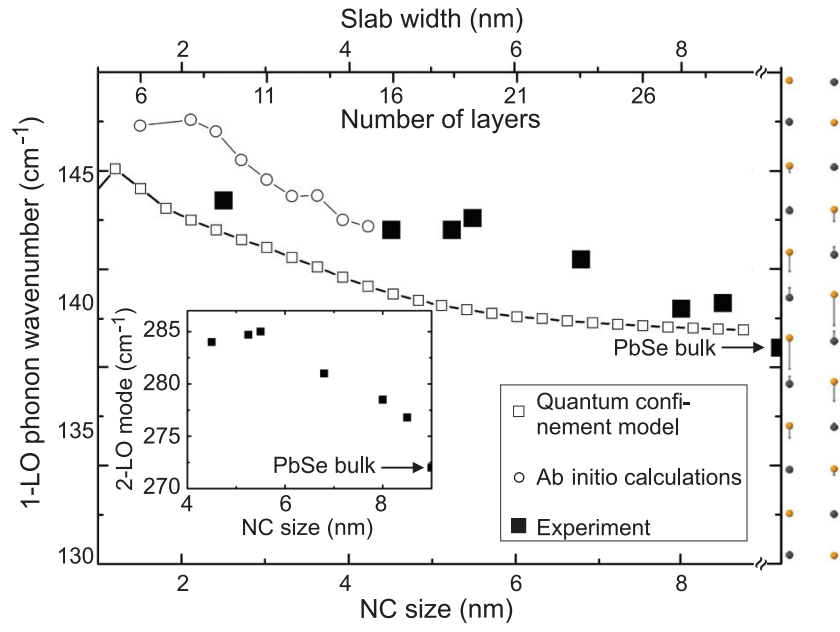

FIG. 4. (Color online) Raman shift of the 1-LO peak as a function of the PbSe nanostructure size. Open circles: calculated frequencies of the 1-LO peak for PbSe (100) slabs with a width varying from 6 layers up to 15 layers. Open squares: 1-LO frequencies for $\mathrm{PbSe}(100)$ slabs according to the quantum-confinement model (extracted from the dispersion in Fig. 5). Filled squares: measured Raman frequencies for films of PbSe NCswith different sizes. Inset: Raman wavenumbers of the 2-LO peak as a function of the PbSe nanocrystal size. The right panel shows the vibrational pattern of the quantum confined LO mode (with polarization perpendicular to the slab) of a 12-layer slab. The double column of $\mathrm{Pb}$ (gray) and $\mathrm{Se}$ (orange) atoms represents the motif of the elementary unit cell of the slab.

of the 1-LO mode, and the heat dissipation might be more or less efficient depending on the separation between neighbors in the nanocrystal film, we assign these fluctuations to inhomogeneities in the compactness of the nanocrystal array and the degree of disorder. On average, we find a standard deviation of $0.85 \mathrm{~cm}^{-1}$ for the peak position on a given sample.

Reiterating the measurements for the other samples, we were able to plot the energy of the 1-LO and 2-LO peaks as a function of the nanocrystal size. Starting with the bulk value of the 1 -LO peak at $136 \mathrm{~cm}^{-1}$, Fig. 4 reveals a blueshift of the 1 -LO peak as the nanocrystal size decreases from 8.5 to $6 \mathrm{~nm}$. Then the peak position of the 1-LO mode fluctuates around $142 \mathrm{~cm}^{-1}$ for smaller nanocrystal sizes. A similar behavior is obtained for the 2-LO peak, indicating that the energy of the LO mode readily varies with the nanocrystal sizes.

\section{RESULTS}

For the modelling of Raman spectroscopy of nanocrystals, three levels of approximation have been used in the past (for an overview see, e.g., Ref. 35): (i) In the "confinement model" one assumes that the restriction of the phonon mode to a nanocrystal of diameter $D$ leads to an uncertainty $\Delta q \approx$ $\pi / D$ in the wave vector of a zone-center optical phonon which in turn leads to a shift and uncertainty (broadening) of its wavenumber. (ii) In the "continuum model" (see, e.g., Refs. 36-38) the vibrations of the spherical (or differently shaped) nanocrystal are obtained by solving the continuous equation of motion which contains as input the bulk phonon dispersion relation in order to take into account the atomic 


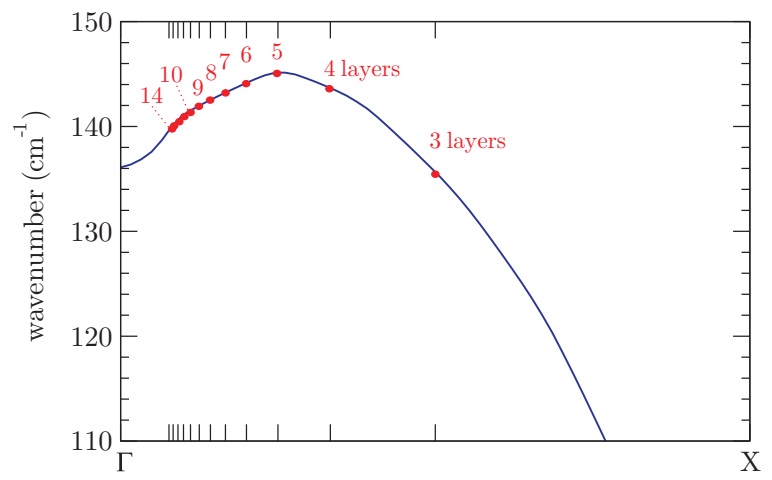

FIG. 5. (Color online) Calculated dispersion of the LO phonon branch between $\Gamma$ and $X$ (Ref. 2). The phonon dispersion has been upscaled by $6 \%$ in order to match the experimental value for the LO phonon at $\Gamma$. The red circles mark the wave vectors and frequencies of the perpendicular LO mode of an $n$-layer slab according to the zone-folding model (see text). For comparison with Fig. 4: the 6-layer slab has a width of $3.45 \mathrm{~nm}$, the 14-layer slab has a width of $8 \mathrm{~nm}$.

character of the vibrations. This equation of motion is coupled with the Poisson equation in order to take into account the coupling of the vibrations to the electric field that may result from the vibrations of a polar material. (iii) Phonons of some nanocrystals [e.g., GaP (Ref. 39)] have been calculated on the atomistic level by diagonalizing the dynamical matrix, which was calculated from force constants and effective charges that were both fitted to the bulk phonon dispersion.

The quantum confinement model has been used to explain the Raman spectra of microcrystalline hexagonal boron nitride $(\mathrm{hBN})$ : with decreasing crystal size, the Raman peak (which is due to the TO phonon in that case) shifts upwards in wavenumber. ${ }^{40}$ The reason comes from the pronounced local minimum at $\Gamma$ of the TO mode, as confirmed by calculations ${ }^{41}$ and measurements ${ }^{42}$ of the phonon dispersion. Thus, a similar analysis may hold for the $\mathrm{LO}$ mode of $\mathrm{PbSe}$ which also displays a minimum at $\Gamma .^{2,10}$ This situation is demonstrated in Fig. 5 where we show the LO mode dispersion in the $\Gamma X$ direction (i.e., for the phonon wave vector $\mathbf{q}$ in the [001] direction). While it is not possible to associate the phonon modes of spherical nanocrystals directly with a bulk mode, this analysis can be done easily for the phonon modes of slabs (2D-systems). The wave vector in the direction perpendicular to the slab surface is quantized. This leads to the mapping of the node free LO mode of an $n$-layer (100) slab to the bulk LO phonon with wave vector $\mathbf{q}_{n}=\mathbf{q}_{\max } /(n-1)$, where $\mathbf{q}_{\max }$ denotes the wave vector at the high-symmetry point $X$ on the boundary of the first Brillouin zone. The wave vectors $\mathbf{q}_{n}$ and the corresponding frequencies are shown by red circles in Fig. 5. In this zone-folding scheme, the LO mode of slabs does indeed blueshift with decreasing number of layers until the maximum is reached for $n=5$. For smaller layers, the frequency should decrease again, but here the limit of the validity of the zone-folding method is reached. The wavenumber difference between bulk and the 5-layer slab is almost $10 \mathrm{~cm}^{-1}$, in good agreement with the experimental data of Fig. 4.

The continuum model was used by Krauss et al. ${ }^{24}$ for the analysis of their Raman data of PbS nanocrystals with diameter of $4 \mathrm{~nm}$. Since it uses as an input the bulk phonon dispersion which displays a dip for the LO mode at $\Gamma{ }^{6}$ the model correctly predicts a blueshift of the Raman peak with respect to the bulk LO model. Thus, both the quantum confinement and the continuum model describe qualitatively correctly the blueshift of the Raman peak with decreasing nanocrystal diameter. Nevertheless, both models do not take into account the changes of the electronic structure (band gap, dielectric screening, effective charges) with decreasing crystal diameter. It would thus be desirable to perform microscopic lattice dynamical calculations (such as in Ref. 39 for $\mathrm{GaP}$ quantum dots). Due to the very peculiar electron and phonon dispersions, a good semiempirical model for the interatomic force constants is not available (and would also not take into account the changes in dielectric screening and effective charges). In principle, one should therefore perform ab initio calculations of the phonons. Since this is currently still not feasible for nanocrystals containing several hundreds or thousands of atoms, we performed ab initio calculations of phonons in a slab geometry which allows studying the effect of a one-dimensional quantum confinement.

We use density functional theory (DFT) in the local density approximation (LDA) as implemented in the code QUANTUM ESPRESSO.$^{43}$ The slabs consist of 6 to 15 layers in the (001) orientation. The slabs are arranged in a periodic supercell with a vacuum of a width of 14 a.u. (before geometry relaxation) between adjacent slabs. Wave functions are expanded in plane-waves with an energy cutoff of $30 \mathrm{Ry}$ and the first Brillouin zone is sampled by a $10 \times 10 \times 2 \mathrm{k}$-point mesh. For $\mathrm{Pb}$, we use a Vanderbilt ultrasoft pseudopotential with the $5 d$ electrons in the valence, and for Se we use a norm-conserving Bachelet-Hamann-Schlüter pseudopotential. For the in-plane lattice constant, we choose $6.124 \AA$, which is the experimental value at room temperature. ${ }^{1}$ In the out-of-plane direction, the geometry is optimized, which leads to a $1.3 \%$ decrease in the nearest neighbor distance and to a surface rumpling $\left(z_{\mathrm{Se}}-z_{\mathrm{Pb}}\right.$, where $z$ denotes the coordinate perpendicular to the layer) of $0.072 \AA$ A.

The phonon frequencies are obtained from the equation

$$
\operatorname{det}\left|\frac{1}{\sqrt{M_{s} M_{t}}} \tilde{C}_{\mathrm{st}}^{\alpha \beta}(\mathbf{q})-\omega^{2}(\mathbf{q})\right|=0 .
$$

The dynamical matrix $\tilde{C}_{\mathrm{st}}^{\alpha \beta}(\mathbf{q})$ corresponds to the change of the force acting on atom $t$ in direction $\beta$ with respect to a displacement of atom $s$ in direction $\alpha$. In Raman spectroscopy, only modes with a wave vector $\mathbf{q} \rightarrow 0$ are excited. The elements of the dynamical matrix are calculated by density functional perturbation theory (DFPT) ${ }^{44,45}$

The vibrational mode that is important for our analysis is the LO mode whose vibrational motion is perpendicular to the slab and whose "envelope" wave function has an antinode in the middle of the slab and vanishes at the surface (see right panel of Fig. 4). This mode is easily discernible by its high infrared intensity because all anions are moving in one direction and the cations oppositely. This is also the reason for the strong Fröhlich coupling which renders this mode Raman active under resonant conditions even though its $A_{2 u}$ symmetry [the point group of the slab is $D_{4 h}(4 / \mathrm{mmm})$ ] renders this mode inactive under nonresonant conditions. Due to the polarity of 
the mode, one has to take into account the macroscopic electric field that accompanies the collective atomic displacements and adds an additional "nonanalytical" term to the dynamical matrix $^{44}$

$$
\tilde{C}_{\mathrm{st}}^{\alpha \beta}(\mathbf{q})={ }^{\mathrm{an}} \tilde{C}_{\mathrm{st}}^{\alpha \beta}(\mathbf{q})+{ }^{\mathrm{na}} \tilde{C}_{\mathrm{st}}^{\alpha \beta}(\mathbf{q}),
$$

with

$$
{ }^{\mathrm{na}} \tilde{C}_{\mathrm{st}}^{\alpha \beta}(\mathbf{q})=\frac{4 \pi}{\Omega} e^{2} \frac{\left(\mathbf{q} \cdot \mathbf{Z}_{s}^{*}\right)_{\alpha}\left(\mathbf{q} \cdot \mathbf{Z}_{s}^{*}\right)_{\beta}}{\mathbf{q} \cdot \varepsilon_{\infty} \cdot \mathbf{q}} .
$$

Here, $\Omega$ is the unit-cell volume. The electronic dielectric tensor $\varepsilon_{\infty}$ and the effective charge tensor $Z_{s}^{*}$ are calculated within DFPT, just as the analytical part of the dynamical matrix ${ }^{\text {an }} \tilde{C}_{\text {st }}^{\alpha \beta}(\mathbf{q})$. The name "nonanalytical" stems from the fact that in the limit $\mathbf{q} \rightarrow 0$, the value of ${ }^{\text {na }} \tilde{C}_{\text {st }}^{\alpha \beta}(\mathbf{q})$ in Eq. (3) depends on the direction in which the limit is taken. In our calculations, the limit is taken in the direction perpendicular to the layers.

The results of our calculations are displayed in Fig. 4 together with the experimental data points and the results from the quantum-confinement model (extracted from Fig. 5). The calculations confirm the experimentally observed trend of increasing LO frequency with decreasing nanocrystal size. For slabs with more than 10 layers, the $a b$ initio results are parallel to the predictions of the quantum-confinement model. The difference of about $3 \mathrm{~cm}^{-1}$ can be attributed to surface effects and to the fact that in the slab calculation we perform a geometry optimization in the perpendicular direction while the (in-plane) lattice constant is the experimental bulk lattice constant. $^{46}$

An important uncertainty in our comparison of calculations and experiment consists in the dielectric environment of the nanocrystals, which can play a role in the coupling of the induced macroscopic electric field with the LO mode. On one hand, in our calculations, we use vacuum (with a dielectric constant of 1) in between the layers. On the other hand, the periodic stacking of PbSe slabs leads to an average macroscopic dielectric constant (in the perpendicular direction) between 4.1 (for the 6-layer slab) and 7.2 (for the 15-layer slab) ${ }^{47}$ and thus to an effective dielectric environment which is probably not very far from the situation of a stacking of nanocrystals separated by layers of organic materials. Increasing the vacuum width between the slabs reduces the average dielectric constant but also reduces the effective charges such that the net influence of the vacuum width in our calculations is rather weak.

Finally, we shall examine the line shape of the peaks. As seen in Fig. 1(c), the peak of the 1-LO mode shows a small shoulder at $125 \mathrm{~cm}^{-1}$ and a broad shoulder around $200 \mathrm{~cm}^{-1}$. Such features are better seen in Fig. 6 that highlights the experimental variation of the peak intensity as a function of the laser power for a sample consisting of nanocrystals and a bulk sample. The shoulder at low frequency, labelled SP, appears for the nanocrystal and bulk samples and has been discussed above as originating from the $\mathrm{Pb}-\mathrm{O}-\mathrm{Pb}$ stretching mode at the surface of the nanocrystals. In contrast to this low-frequency component, the broad shoulder at high frequency is not always visible. Only for the highest laser powers, the peak of the 1-LO mode becomes really asymmetric. As this asymmetry is also seen for the bulk sample [Fig. 6(b)], we rule out any confinement effect as the cause of this line shape modification.
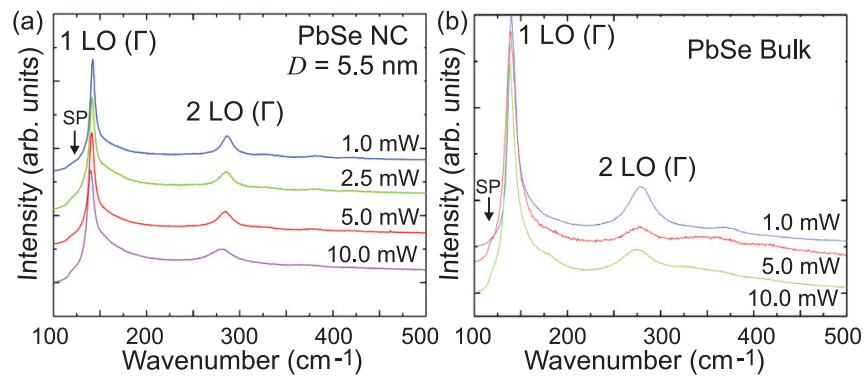

FIG. 6. (Color online) Raman spectra of (a) a 5.5-nm PbSe nanocrystal film and (b) a bulk PbSe sample measured for different laser powers.

Interestingly, we note that the change of the line shape takes place with a reduction of the peak intensity of the 2-LO mode. Measurement of the ratio between the peak intensity of the 2 -LO mode yields a reproducible value, around 0.65 , when the spectra at the lowest and highest laser powers are compared. Since this ratio reflects the electron-phonon coupling strength, a reproducible decrease of the peak intensity indicates a stronger screening of the electron-LO-phonon interaction, consistent with a higher density of free charge carriers in the samples as the excitation power increases. Therefore, we suspect the asymmetry to correspond to a Fano effect ${ }^{48}$ rather than a nonadiabatic effect. ${ }^{38}$ Indeed, the interaction of electronic Raman transitions with discrete optical phonons results in Fano interference, and severe asymmetries have been found for heavily doped semiconductor crystals ${ }^{48,49}$ and nanostructures. ${ }^{50}$ In the case of PbSe nanocrystals, due to the eightfold degeneracy of the lowest conduction and valence band levels that can be slightly lifted up under confinement ${ }^{51}$ and the high polarizability of the $p$-like orbitals, ${ }^{32}$ we think that the intraband electronic transitions between different valleys could be resonant with the LO phonon mode ${ }^{52}$ and give rive rise to the asymmetric line shape.

\section{CONCLUSION}

The measured ${ }^{6,12}$ and calculated ${ }^{2}$ anomaly of the zonecenter LO phonon mode in the dispersion of bulk PbSe manifests itself also in the diameter dependence of the Raman spectra of PbSe nanocrystals. Contrary to most nanocrystalline materials, where the Raman active phonon modes shift down in frequency, we observe a blueshift of the Raman peak with decreasing nanocrystal diameter. This behavior can be qualitatively understood in terms of quantum confinement of the LO mode because the phonon dispersion has a dip at the zone center. Decreasing the crystal diameter means increasing the value of the quantized wave vector and thus leads to an increase of the frequency. This qualitative behavior is confirmed by our ab initio phonon calculations on $\mathrm{PbSe}$ slabs of various widths. These calculations take into account the changes in the electronic structure (band-gap opening) and the dielectric confinement. In addition to optical absorption spectroscopy, Raman spectroscopy is thus a suitable tool for the diameter determination of PbSe nanocrystals. Due to the similar electronic and vibrational structure, we expect that the same conclusions also hold for $\mathrm{PbS}$ and $\mathrm{PbTe}$ nanocrystals. 


\section{ACKNOWLEDGMENTS}

We acknowledge support from the EU Seventh Framework Program (EU-FP7 ITN Herodot, Grant No. PITNGA-2008-214954). Calculations were performed at the IDRIS supercomputing center, Orsay (Proj. No. 091827).
Technion laboratory was supported by the EU-FP7 SANS (Self-Assembled Nanostructure System) project, Israel Science Foundation Project No. 1009/07 and 1425/04, and USA-Israel Binational Science Foundation (Project No. 2006-225).
*Corresponding author: ludger.wirtz@uni.lu

${ }^{1}$ Semiconductors: Group IV Elements, IV-IV and III-IV Compounds, Landolt-Börnstein, New Series, Group III, Vol. 41, Pt. A, edited by O. Madelung, U. Rössler, and M. Schulz (Springer-Verlag, Berlin, 2005).

${ }^{2}$ O. Kilian, G. Allan, and L. Wirtz, Phys. Rev. B 80, 245208 (2009).

${ }^{3}$ A. Svane, N. E. Christensen, M. Cardona, A. N. Chantis, M. van Schilfgaarde, and T. Kotani, Phys. Rev. B 81, 245120 (2010).

${ }^{4}$ K. Hummer, A. Grüneis, and G. Kresse, Phys. Rev. B 75, 195211 (2007).

${ }^{5}$ W. Jantsch, A. Bussmann-Holder, H. Bilz, and P. Vogl, Dynamical Properties of IV-VI Compounds, Springer Tracts in Modern Physics Vol. 99 (Springer, Berlin, Heidelberg, 1983).

${ }^{6}$ M. M. Elcombe, Proc. Roy Soc. A 300, 210 (1967).

${ }^{7}$ E. Burstein and A. Pinczuk, in Physics of Optoelectronic Materials, edited by W. A. Albers (Plenum Press, New York, 1971).

${ }^{8}$ W. Cochran, R. A. Cowley, G. Dolling, and M. M. Elcombe, Proc. Roy Soc. A 293, 433 (1966).

${ }^{9}$ Indeed, the calculated TO frequency becomes imaginary when the lattice constant is increased.

${ }^{10}$ O. B. Maksimenko and A. S. Mishchenko, J. Phys.: Condens. Matter. 9, 5561 (1997).

${ }^{11}$ The near Kohn anomaly turns into a real Kohn anomaly when PbS is compressed by $1.8 \%$ and becomes quasimetallic.

${ }^{12}$ P. R. Vijayraghavan, S. K. Sinha, and P. K. Iyengar, Proc. Nucl. Phys. Solid State Phys. (India) 16C, 208 (1973).

${ }^{13}$ L. K. Vodop'yanov, I. V. Kucherenko, A. P. Shotov, and R. Sherm, JETP Lett. 21, 101 (1978).

${ }^{14}$ B. Minceva-Sukarova, M. Najdoski, I. Grozdanov, and C. J. Chunnilall, J. Mol. Struct. 410-411, 267 (1997).

${ }^{15}$ A.-L. Yang, H.-Z. Wu, Z.-F. Li, D.-J. Qiu, Y. Chang, J.-F. Li, P. J. McCann, and X. M. Fang, Chin. Phys. Lett. 17, 606 (2000).

${ }^{16}$ R. N. Hall and J. H. Racette, J. Appl. Phys. 32, 2078 (1961).

${ }^{17}$ L. Brillson and E. Burstein, Phys. Rev. Lett. 27, 808 (1971).

${ }^{18}$ S. V. Ovsyannikov, Y. S. Ponosov, V. V. Shchennikov, and V. E. Mogilenskikh, Phys. Stat. Sol. C 1, 3110 (2004).

${ }^{19}$ G. D. Smith, S. Firth, R. J. H. Clark, and M. Cardona, J. Appl. Phys. 92, 4375 (2002).

${ }^{20}$ F. W. Wise, Acc. Chem. Res. 33, 773 (2000).

${ }^{21}$ R. Koole, G. Allan, C. Delerue, A. Meijerink, D. Vanmaekelbergh, and A. J. Houtepen, Small 4, 127 (2008).

${ }^{22}$ I. Moreels, K. Lambert, D. Smeets, D. De Muynck, T. Nollet, J. C. Martins, F. Vanhaecke, A. Vantomme, C. Delerue, G. Allan, and Zeger Hens, ACS Nano 3, 3023 (2009).

${ }^{23}$ H. Fu and S.-W. T. Sang, Nanoscale 4, 2187 (2012).

${ }^{24}$ T. D. Krauss, F. W. Wise, and D. B. Tanner, Phys. Rev. Lett. 76, 1376 (1996).

${ }^{25}$ K. K. Nanda, S. N. Sahu, R. K. Soni, and S. Tripathy, Phys. Rev. B 58, 15405 (1998).
${ }^{26}$ F. S. Manciu, Y. Sahoo, F. Carreto, and P. N. Prasad, J. Raman Spectrosc. 39, 1135 (2008).

${ }^{27}$ A. Kigel, M. Brumer, G. Maikov, A. Sashchiuk, and E. Lifshitz, Superlattices Micro. 46, 272 (2009).

${ }^{28}$ I. Moreels, B. Fritzinger, J. C. Martins, and Z. Hens, J. Am. Chem. Soc. 130, 15081 (2008).

${ }^{29}$ E. A. Albanesi, C. M. I. Okoye, C. O. Rodriguez, E. L. Peltzer y Blanca, and A. G. Petukhov, Phys. Rev. B 61, 16589 (2000).

${ }^{30}$ C. R. Bealing, W. J. Baumgardner, J. J. Choi, T. Hanrath, and R. G. Hennig, ACS Nano 6, 2118 (2012).

${ }^{31}$ K.-S. Cho, D. V. Talapin, W. Gaschler, and C. B. Murray, J. Am. Chem. Soc. 127, 7140 (2005).

${ }^{32}$ G. Allan and C. Delerue, Phys. Rev. B 70, 245321 (2004).

${ }^{33}$ S. Sahoo and A. K. Arora, J. Phys. Chem. B 114, 4199 (2010).

${ }^{34}$ J. L. Blackburn, H. Chappell, J. M. Luther, A. J. Nozik, and J. C. Johnson, J. Phys. Chem. Lett. 2, 599 (2011).

${ }^{35}$ A. K. Arora, M. Rajalakshmi, T. R. Ravindran, and V. Sivasubramanian, J. Raman Spectrosc. 38, 604 (2007).

${ }^{36}$ E. Roca, C. Trallero-Giner, and M. Cardona, Phys. Rev. B 49, 13704 (1994).

${ }^{37}$ A. G. Rolo and M. I. Vasilevskiy, J. Raman Spectrosc. 38, 618 (2007).

${ }^{38}$ E. P. Pokatilov, S. N. Klimin, V. M. Fomin, J. T. Devreese, and F. W. Wise, Phys. Rev. B 65, 075316 (2002).

${ }^{39}$ H. Fu, V. Ozolins, and A. Zunger, Phys. Rev. B 59, 2881 (1999).

${ }^{40}$ R. J. Nemanich, S. A. Solin, and R. M. Martin, Phys. Rev. B 23, 6348 (1981).

${ }^{41}$ G. Kern, G. Kresse, and J. Hafner, Phys. Rev. B 59, 8551 (1999).

${ }^{42}$ J. Serrano, A. Bosak, R. Arenal, M. Krisch, K. Watanabe, T. Taniguchi, H. Kanda, A. Rubio, and L. Wirtz, Phys. Rev. Lett. 98, 095503 (2007).

${ }^{43}$ P. Giannozzi, S. Baroni, N. Bonini, M. Calandra, R. Car, C. Cavazzoni, D. Ceresoli, G. L. Chiarotti, M. Cococcioni, I. Dabo, A. Dal Corso, S. de Gironcoli, S. Fabris, G. Fratesi, R. Gebauer, U. Gerstmann, C. Gougoussis, A. Kokalj, M. Lazzeri, L. Martin-Samos, N. Marzari, F. Mauri, R. Mazzarello, S. Paolini, A. Pasquarello, L. Paulatto, C. Sbraccia, S. Scandolo, G. Sclauzero, A. P. Seitsonen, A. Smogunov, P. Umari, and R. M. Wentzcovitch, J. Phys.: Condens. Matter 21, 395502 (2009).

${ }^{44}$ S. Baroni, P. Giannozzi, and A. Testa, Phys. Rev. Lett. 58, 1861 (1987); X. Gonze and C. Lee, Phys. Rev. B 55, 10355 (1997); S. Baroni, S. de Gironcoli, A. Dal Corso, and P. Giannozzi, Rev. Mod. Phys. 73, 515 (2001).

${ }^{45} \mathrm{~A}$ relevant question for the calculation of phonon frequencies in lead chalcogenides is the inclusion of spin-orbit coupling (SOC). Romero et al. [A. H. Romero, M. Cardona, R. K. Kremer, R. Lauck, G. Siegle, J. Serrano, and X. C. Gonze, Phys. Rev. B 78, 224302 (2008)] have shown that SOC causes a slight softening of all the phonon branches. However, for the LO mode around $\Gamma$, 
LDA calculations with SOC give accidentally a worse agreement with experimental data. This is due to the fact that SOC reduces the band gap and leads to an underestimation (or even an inversion) of the band gap. Since the very small band gap of lead chalcogenides gives rise to the anomalous dip of the LO mode [Kilian et al. (Ref. 2)], an underestimation of the gap will lead to an exaggerated dip of the LO mode. For a completely correct calculation of the LO mode at $\Gamma$, one would have to correct the band gap of lead chalcogenides with the $G W$ method on top of LDA + SOC or by using a hybrid functional together with SOC [as has been done in Hummer et al. (Ref. 4)]. Calculating phonons in that context has not been achieved yet. Thus, the best compromise to date is the use of LDA without SOC, which (by cancellation of errors) yields a reasonable band gap of all three lead chalcogenides and thus reproduces quantitatively the anomalous phonon dip of the LO mode at $\Gamma$.

${ }^{46}$ The fact that the calculated frequencies are higher than the measured ones is due to the fact that the local density approximation tends to overbind, i.e., it underestimates the bond length (overbinding) and thus overestimates the phonon frequencies when a geometry optimization is performed.

${ }^{47}$ For details of the dependence of the dielectric constant on the slab width and on the relation between the average macroscopic dielectric constant and the dielectric constant in the interior of the slabs, we refer the reader to the discussion in I. Moreels, G. Allan, B. De Geyter, L. Wirtz, C. Delerue, and Z. Hens, Phys. Rev. B 81, 235319 (2010).

${ }^{48}$ F. Cerdeira, T. A. Fjeldly, and M. Cardona, Phys. Rev. B 8, 4734 (1973).

${ }^{49}$ D. Olego and M. Cardona, Phys. Rev. B 24, 7217 (1981).

${ }^{50}$ T. Kawashima, G. Imamura, T. Saitoh, K. Komori, M. Fujii, and S. Hayashi, J. Phys. Chem. C 111, 15160 (2007).

${ }^{51}$ K. Overgaag, D. Vanmaekelbergh, P. Liljeroth, G. Mahieu, B. Grandidier, C. Delerue, and G. Allan, J. Chem. Phys. 131, 224510 (2009).

${ }^{52}$ R. Ulbricht, J. J. H. Pijpers, E. Groeneveld, R. Koole, C. de Mello Donega, D. Vanmaekelbergh, C. Delerue, G. Allan, and M. Bonn, Nano. Lett. 12, 4937 (2012). 